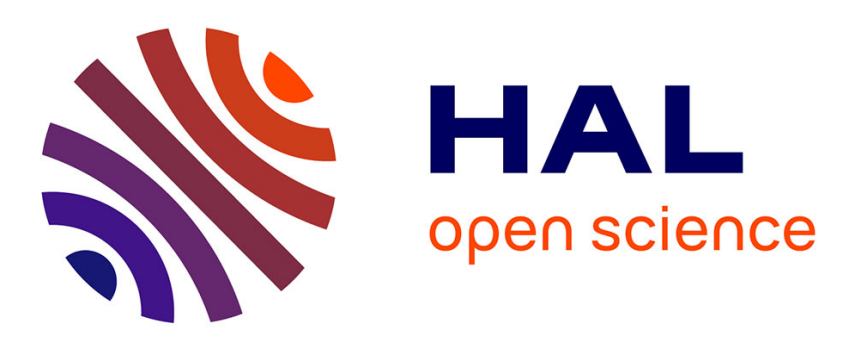

\title{
A Badly Calibrated Camera in Ego-Motion Estimation, Propagation of Uncertainty
}

\author{
Tomas Svoboda, Peter Sturm
}

\section{To cite this version:}

Tomas Svoboda, Peter Sturm. A Badly Calibrated Camera in Ego-Motion Estimation, Propagation of Uncertainty. 7th International Conference on Computer Analysis of Images and Patterns (CAIP '97), Sep 1997, Kiel, Germany. pp.183-190, 10.1007/3-540-63460-6_116 . inria-00590083

\section{HAL Id: inria-00590083 https://hal.inria.fr/inria-00590083}

Submitted on 3 May 2011

HAL is a multi-disciplinary open access archive for the deposit and dissemination of scientific research documents, whether they are published or not. The documents may come from teaching and research institutions in France or abroad, or from public or private research centers.
L'archive ouverte pluridisciplinaire HAL, est destinée au dépôt et à la diffusion de documents scientifiques de niveau recherche, publiés ou non, émanant des établissements d'enseignement et de recherche français ou étrangers, des laboratoires publics ou privés. 


\title{
A Badly Calibrated Camera in Ego-Motion Estimation, Propagation of Uncertainty
}

\author{
Tomáš Svoboda and Peter Sturm \\ svoboda@vision.felk.cvut.cz
}

\section{Reference}

[1] T. Svoboda and P. Sturm. A badly calibrated camera in ego-motion estimation, propagation of uncertainty. Submitted to CAIP'97 conference, January 1997.

This publication can be obtained via anonymous ftp from ftp://cmp.felk.cvut.cz/pub/cvl/ARTICLES/SVOBODA/caip97submitted.ps.gz

Czech Technical University, Faculty of Electrical Engineering Department of Control Engineering, Center for Machine Perception, Computer Vision Laboratory 12135 Prague 2, Karlovo náměstí 13, Czech Republic, FAX +42 2290159, phone +42 224357465 


\title{
CAIP'97 submission - cover page \\ A Badly Calibrated Camera in Ego-Motion Estimation, Propagation of Uncertainty
}

\author{
Tomáš Svoboda \\ Center for Machine Perception \\ Czech Technical University \\ Karlovo nám. 13 \\ CZ 121-35 Praha 2, Czech Republic \\ tel. $+42-2-24.35 .74 .58^{1}$ \\ fax $+42-2-29.01 .59^{2}$ \\ svoboda@vision.felk.cvut.cz
}

and Peter Sturm

GRAVIR-IMAG

INRIA Rhône-Alpes

655, avenue de l'Europe

38330 Monbonnot, France

tel. $+33-4.76 .61 .52 .32$

fax $+33-4.76 .61 .52 .52$

peter.sturm@inrialpes.fr

\begin{abstract}
This paper deals with the ego-motion estimation (motion of the camera) from two views. When we want to estimate the ego-motion we have to find correspondences and we need a calibrated camera. In this paper we solve the problem how to propagate known camera calibration errors into the uncertainty of the motion parameters. We present the linear estimate of the uncertainty of the motion parameters based on the uncertainty in the calibration parameters. We show that the linear estimate of the motion parameters uncertainty is very stable and useful up to $20 \%$ noise in camera calibration even with a noise in correspondences.
\end{abstract}

\section{Which category is most appropriate for the submission?}

This work best fits to the topic motion and stereo.

\footnotetext{
${ }^{1}+420-2-24.35 .74 .58$ since 1 March 1997

2+420-2-29.01.59 since 1 March 1997
} 


\title{
CAIP'97 submission - summary page \\ A Badly Calibrated Camera in Ego-Motion Estimation, Propagation of Uncertainty
}

\author{
Tomáš Svoboda Peter Sturm
}

\section{What is the original contribution of this work?}

This work presents a linear algorithm to estimate of the uncertainty in the motion parameters when the covariances of the camera calibration is known. We decompose the whole ego-motion algorithm into several substeps and we propagate the covariances throughout the estimation process. We have found several worthwhile relationships between noise in calibration parameters and allied noise in motion parameters. We have found out that linear estimate is stable and trustworthy even with noise in correspondences. This linear estimate is useful up to $20 \%$ noise in calibration parameters.

\section{What is the most closely related work by others and how does this work differ?}

Our work is closely related to [2] where an error analysis of the ego-motion algorithm is proposed. However, only noise in correspondences is considered. The camera calibration is supposed to be done correctly. Flourou and Mohr in [1] used the statistic approach to find out the relationships between errors in the camera calibration and the errors in the reconstruction. Nevertheless only errors in the reconstruction is investigated and only a statistic approach has been used. We present an on-line linear algorithm to estimate errors. So our algorithm can be used to predict the errors in ego-motion parameters resp. their reliability before the incident motion is estimated.

\section{References}

[1] Giannoula Florou and Roger Mohr. What accuracy for 3D measurement with cameras? In International Conference on Pattern Recognition 1996, pages 354-358, Los Alamitos, California, August 1996. IEEE Computer Society Press.

[2] Juyang Weng, Thomas S. Huang, and Ahuja Narendra. Motion and structure from two perspective views: Algorithms, error analysis, and error estimation. IEEE Transactions on Pattern Analysis and Machine Inteligence, 11(5):451-476, May 1989. 


\title{
A Badly Calibrated Camera in Ego-Motion Estimation, Propagation of Uncertainty
}

\begin{abstract}
This paper deals with the ego-motion estimation (motion of the camera) from two views. When we want to estimate the ego-motion we have to find correspondences and we need a calibrated camera. In this paper we solve the problem how to propagate known camera calibration errors into the uncertainty of the motion parameters. We present the linear estimate of the uncertainty of the motion parameters based on the uncertainty in the calibration parameters. We show that the linear estimate of the motion parameters uncertainty is very stable and useful up to $20 \%$ noise in camera calibration even with a noise in correspondences.
\end{abstract}

\section{Introduction}

Let us assume to have two images captured by the same camera from two different viewpoints. When we find at least eight corresponding points and when we know the calibration parameters of the camera, the camera motion can be estimated up to a similarity. The similarity reconstruction of the scene can be done, too. This problem has been solved over years $[7,13]$. When the camera calibration is not known, other algorithms have to be used. Since we have only two images we can only establish the epipolar geometry (Luong et al. in [8]) and we can do only projective reconstruction. If we want to calibrate the camera we can use some of the many methods developed for the off-line camera calibration. Tsai did this in $[12]$.

Having more images, at least three, we can employ the algorithms for the camera selfcalibration. This theory was presented by Maybank and Faugeras in [9] or, when the camera undergoes planar motion, we can use the algorithm developed by Armstrong et al. in [1]. Hartley has presented an iterative algorithm for Euclidean reconstruction from many uncalibrated views in [3].

In this paper we deal with two views and a roughly calibrated camera. We use a linear method intended for a calibrated camera to estimate the ego-motion. Because of noise in the estimation process, an error analysis is needed. There are two sources of errors: (a) noise in correspondences (b) noise in the calibration parameters. Weng [13] studied the influence of the noise on the motion parameters. Florou and Mohr [2] used the statistic approach to study reconstruction errors with the calibration parameters. In our paper we present a linear algorithm for the estimate of the validity of the motion parameters based on the uncertainty in the calibration parameters. 


\section{Fundamentals}

\section{$2.1 \quad$ Notation}

Vectors will be denoted by bold characters. There will be two types of vectors: (a) normal, in conventional sense, this type of vectors will be denoted by small bold characters $\mathbf{u}=\left[u_{1}, u_{2}, \ldots, u_{n}\right]^{T}$, (b) column vectors which arise from the elements of the matrices, for instance the $3 \times 3$ matrix $E$, rearranged $9 \times 1$ vector, will be denoted by the capital bold character $\mathbf{E}$. The matrices are indicated by capital italics such as $E$. This notation will stand also in the subscripts. The sign $\triangleq$ we use to define new variables when the variable to be defined is obvious. The sign $\cong$ denotes the approximate equality.

\subsection{The camera model and the camera calibration}

We use the classical pinhole camera model. There are three coordinate systems as it is illustrated in Figure 1. The world coordinate system $\left(W, x_{w}, y_{w}, z_{w}\right)$, the camera standard

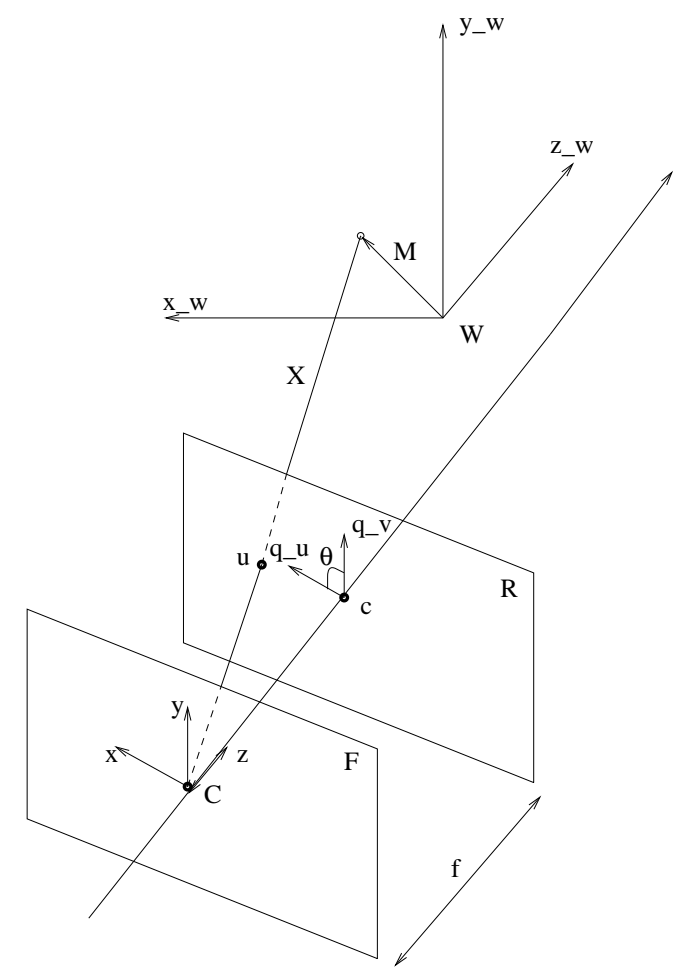

Figure 1: The pinhole camera, retinal (R) and focal (F) plane and three coordinate systems. coordinate system $(C, x, y, z)$ and the coordinate system for the retinal plane $\left(c, q_{u}, q_{v}\right)$. Let us introduce the transformations among them:

$$
\left[\begin{array}{l}
x \\
y \\
z
\end{array}\right]=[R \mid-R \mathbf{t}]\left[\begin{array}{c}
x_{w} \\
y_{w} \\
z_{w} \\
1
\end{array}\right]
$$


The rotation matrix $R$ and the translation vector $\mathbf{t}$ characterize the orientation and the position of the camera with respect to the world coordinate system. We introduce the normalized image coordinates as

$$
\mathbf{u}=\left[\begin{array}{l}
u \\
v \\
1
\end{array}\right]=\left[\begin{array}{c}
f \frac{x}{z} \\
f \frac{y}{z} \\
1
\end{array}\right]
$$

where $f$ is the focal length of the camera. However, when we localize points in the image, we get the coordinates in coordinate system of the retinal plane in pixels, $\mathbf{q}=\left[q_{u}, q_{v}, 1\right]^{T}$. What is the relationship between those pixel coordinates and the normalized coordinates $\mathbf{u}$ ? Introducing the calibration matrix $K$, we can write

$$
\left[\begin{array}{c}
\frac{u}{f} \\
\frac{v}{f} \\
1
\end{array}\right]=\left[\begin{array}{c}
\frac{x}{z} \\
\frac{y}{z} \\
1
\end{array}\right]=\left[\begin{array}{ccc}
f k_{u} & -f k_{u} \cot (\theta) & q_{u 0} \\
0 & \frac{f k_{v}}{\sin (\theta)} & q_{v 0} \\
0 & 0 & 1
\end{array}\right]^{-1}\left[\begin{array}{c}
q_{u} \\
q_{v} \\
1
\end{array}\right] \triangleq K^{-1} \mathbf{q}
$$

where $f$ is the focal length of the camera, $1 / k_{u}$, resp. $1 / k_{v}$, is the horizontal, resp. vertical, size of the pixels in length units, $q_{u 0}$ and $q_{v 0}$ are pixel coordinates of the principal point. The principal point is the intersection of the optical axis with the retinal plane, in Figure 1 it is denoted by "c". The angle $\theta$ is the angle in the retinal coordinate system. The parameters $k_{u}, k_{v}$ have to be considered together with $f$. The skew angle $\theta$ is usually supposed to be very close to $\pi / 2$, which is a valid approximation in practice. Thus the matrix $K$ can be rewritten in a simpler form as

$$
K=\left[\begin{array}{ccc}
\alpha_{u} & 0 & q_{u 0} \\
0 & \alpha_{v} & q_{v 0} \\
0 & 0 & 1
\end{array}\right]
$$

We can describe the introduced calibration parameters, $\alpha_{u}$, resp. $\alpha_{v}$, as the focal length expressed in the horizontal resp. vertical pixel size. In this paper we assume that the matrix $K$ is in its simpler form (4). The above parameters are sometimes called intrinsic parameters of the camera, whereas $R$ and $\mathbf{t}$ are often titled extrinsic parameters of the camera.

\subsection{Ego-motion from correspondences}

Having at least eight correspondences from two different viewpoints, resp. their pixel coordinates, the epipolar geometry can be established. Both images are supposed to be taken by the same camera. Let the displacement between these position be determined by translation vector $\mathbf{t}$ and rotation matrix $R$. Because of the space restriction the reader is referred to [8] for more information about epipolar geometry. We revoke only the fundamental matrix which can be estimated by solving the set of linear equations

$$
\mathbf{q}_{i}^{\prime T} F \mathbf{q}_{i}=0, \text { where } i=1 \ldots N,
$$

where $\mathbf{q}_{i}^{\prime}$ resp. $\mathbf{q}_{i}$ are the pixel coordinates of correspondences and $N$ is the number of correspondences. By exploiting the calibration matrix (3) we can compute the essential matrix as

$$
E=K^{T} F K
$$


The essential matrix can be also expressed as a product $E=R S$, where $R$ is a rotation matrix and $S$ is an antisymmetric matrix related to $\mathbf{t}$ by the formula

$$
S=\left[\begin{array}{ccc}
0 & -t_{z} & t_{y} \\
t_{z} & 0 & -t_{x} \\
-t_{y} & t_{x} & 0
\end{array}\right]
$$

It is obvious that the essential matrix contains the motion parameters. To obtain $R$ and $\mathbf{t}$ from this essential matrix we use the method by Hartley [4]. Just the essence of it is given here. If $E$ is estimated correctly, it has rank 2 and its nonzero singular values are equal. Using the singular value decomposition we can factorize $E$ as

$$
E=U D V^{T},
$$

where $U$ and $V$ are orthonormal matrices and $D$ is the diagonal matrix containing singular values of $E$. The rotation matrix $R$ and the matrix $S$ are computed by

$$
\begin{aligned}
& S=V Z V^{T}, \\
& R=U Y V^{T} \text { or } \mathrm{UY}^{\mathrm{T}} \mathrm{V}^{\mathrm{T}},
\end{aligned}
$$

where

$$
Z=\left[\begin{array}{ccc}
0 & -1 & 0 \\
1 & 0 & 0 \\
0 & 0 & 0
\end{array}\right] \text { and } Y=\left[\begin{array}{ccc}
0 & 1 & 0 \\
-1 & 0 & 0 \\
0 & 0 & 1
\end{array}\right]
$$

It is evident from the equation (8) that the eigenspace analysis of a noisy matrix will be necessary.

\section{Propagation of uncertainty}

Let us suppose that we know the characteristics of the calibration parameters, i.e. the calibration matrix, the mean, $\bar{K}$, and their covariance matrix, $C_{K}$. Now we want to propagate the information in $C_{K}$ through the whole estimation process. The desired results are covariance matrices of the motion parameters, i.e. $C_{\mathbf{t}}$ for the translation vector, resp. $C_{\mathcal{E}}$ for the Euler angles.

\subsection{Propagation in few equations}

Let us consider the whole estimation process as a general function $f$. Using this notation the estimation procedure can be rewritten as

$$
[\mathcal{E}, \mathbf{t}]=f(K, F)
$$

where $\mathcal{E}$ denotes the Euler angles, which characterize the camera rotation. Then we can compute the means of the $[\mathcal{E}, \mathbf{t}]$ and their covariance matrices as

$$
\begin{aligned}
{[\overline{\mathcal{E}}, \overline{\mathbf{t}}] } & =f(\bar{K}, \bar{F}) \\
C_{\mathcal{E} \mathbf{t}} & =J_{f} C_{K F} J_{f}^{T}
\end{aligned}
$$


where $J_{f}$ denotes Jacobian matrix computed by

$$
J_{f}=\frac{\partial f(K, F)}{\partial K, F}, \text { at }[K, F]=[\bar{K}, \bar{F}]
$$

It looks that this can easily be done, however the function $f$ is very complicated and nonlinear. We will describe the propagation in the individual steps.

\subsection{Covariance of the essential matrix}

The relationship between $K$ and $E$ is determined by the equation (6). Let us suppose that fundamental matrix $F$ is correctly estimated. The calibration matrix has the mean $\bar{K}$ and the covariance matrix $C_{K}$. To compute $\bar{E}$ and $C_{E}$ the equation $(13,14)$ are used.

$$
\begin{aligned}
\bar{E} & =\bar{K}^{T} F \bar{K}, \\
C_{E} & =\frac{\partial\left(K^{T} F K\right)}{\partial K_{i i}}, \text { at } K=\bar{K} .
\end{aligned}
$$

\subsection{Eigenvalues and eigenvectors of a noisy matrix}

The way how to estimate motion from $E$ has been described in equations (8-10). From these equations follows that the eigenspace analysis of a noisy matrix is needed. Using equation (8) it can be easily verified that

$$
\begin{aligned}
& E E^{T}=U D^{2} U^{T}, \\
& E^{T} E=V D^{2} V^{T} .
\end{aligned}
$$

The columns of $U$ are eigenvectors of $E E^{T}$, resp. the columns of $V$ are eigenvectors of $E^{T} E$ [6]. Both $E E^{T}$ and $E^{T} E$ are $3 \times 3$ symmetric matrices. The diagonal matrix D contains singular values of $E$, or $D^{2}=\operatorname{diag}\left(\lambda_{1}, \lambda_{2}, \lambda_{3}\right)$, where $\lambda_{i}$ are eigenvalues of $E E^{T}$. Since the eigenvalue problem of a symmetric matrix is always well conditioned [14], it is reasonable to assume that the singular values of $E$ vary little with variations in $E$. However the same can not be said about the eigenvectors which are actually the columns of $U$, resp. $V$. Let us express the eigenvectors in the matrix $U$

$$
U=\left[\mathbf{u}_{1}, \mathbf{u}_{2}, \mathbf{u}_{3}\right]
$$

where $\mathbf{u}_{1}$ is an eigenvector associated with $\lambda_{1}$. The perturbed matrix $E E^{T}(\epsilon)$ can be written as

$$
E E^{T}(\epsilon)=\left(E+\Delta_{E}\right)\left(E^{T}+\Delta_{E}^{T}\right)=E E^{T}+\Delta_{E E^{T}},
$$

where $\Delta_{E E^{T}}$ is the linear part of the error,

$$
\Delta_{E E^{T}} \cong \Delta_{E} E^{T}+E \Delta_{E}^{T}
$$

Let $\mathbf{u}_{\mathbf{1}}(\epsilon)$ be the eigenvector of the perturbed matrix $E E^{T}(\epsilon)$ associated with the perturbed eigenvalue $\lambda_{1}(\epsilon)$. Then $\mathbf{u}_{\mathbf{1}}(\epsilon)$ can be written as

$$
\mathbf{u}_{\mathbf{1}}(\epsilon)=\mathbf{u}_{\mathbf{1}}+\delta_{\mathbf{u}_{\mathbf{1}}}
$$


with $\delta_{\mathbf{u}_{1}} \subset \operatorname{span}$ of $\left\{\mathbf{u}_{\mathbf{2}}, \mathbf{u}_{\mathbf{3}}\right\}$. Letting $\epsilon$ be the maximum absolute value of the elements $\Delta_{E E^{T}}$, we have

$$
\Delta_{E E^{T}}=\epsilon B
$$

with $b_{i j}=\delta_{E E_{i, j}^{T}} / \epsilon$. For a sufficiently small $\epsilon$, the perturbation of $\lambda_{1}$ can be expressed by a convergent series

$$
\lambda_{1}(\epsilon)-\lambda_{1} \stackrel{\unrhd}{=} \delta_{\lambda_{1}}=p_{1} \epsilon+p_{2} \epsilon^{2}+p_{3} \epsilon^{3}+\cdots
$$

and similarly the perturbation vector $\delta_{\mathbf{u}_{1}}$ can be expressed by a convergent vector series in the span of $U_{2}=\left[\mathbf{u}_{\mathbf{2}}, \mathbf{u}_{\mathbf{3}}\right]$. For a sufficiently small $\epsilon$, there exist 2-dimensional vectors $\mathbf{g}_{1}, \mathbf{g}_{2}, \ldots$ such that

$$
\delta_{\mathbf{u}_{1}}=\epsilon U_{2} \mathbf{g}_{\mathbf{1}}+\epsilon^{2} U_{2} \mathbf{g}_{\mathbf{2}}+\cdots
$$

If we consider just the linear term in equations (24) and (25), then after a derivation [14], we have for the eigenvalue

$$
\delta_{\lambda_{1}} \cong \mathbf{u}_{\mathbf{1}}^{T} \Delta_{E E^{T}} \mathbf{u}_{\mathbf{1}}
$$

and the eigenvectors satisfy

$$
\delta_{\mathbf{u}_{1}} \cong U \Delta_{1} U^{T} \Delta_{E E^{T}} \mathbf{u}_{\mathbf{1}}
$$

where $\Delta_{1}$ is

$$
\Delta_{1}=\operatorname{diag}\left\{0,\left(\lambda_{1}-\lambda_{2}\right)^{-1},\left(\lambda_{1}-\lambda_{3}\right)^{-1}\right\} .
$$

The proof of equations $(26,27)$ can be found in the appendix of [13], or better, together with more theoretical background in [14]. However, it follows, from the equation (28), that the estimate of the errors in the eigenvector will be unstable when $\lambda_{1}$ is very close to $\lambda_{2}$ what is indeed true. The high estimate of the error is caused by an ambiguity of the choice of the eigenvectors. The matrix $E E^{T}$ is symmetric, so it has linear divisors, the perturbed eigenvectors of $E E^{T}(\epsilon)$ belonging to the equal eigenvalues can be expressed as the linear combination of the noise free eigenvectors

$$
\begin{aligned}
\mathbf{u}_{1}(\epsilon) & =\mathbf{u}_{1}+k(\epsilon) \mathbf{u}_{2}+\alpha_{3}(\epsilon) \mathbf{u}_{3}, \text { or } \\
& =k(\epsilon) \mathbf{u}_{1}+\mathbf{u}_{2}+\alpha_{3}(\epsilon) \mathbf{u}_{3} .
\end{aligned}
$$

The same linear combinations also hold for $\mathbf{u}_{2}(\epsilon)$. Both of these vectors are indeed eigenvectors of $E E^{T}(\epsilon)$. Because of the space restriction the proof is omitted here. The reader is referred to [14]. So, for practical purposes, we rewrite equation (27) as

$$
\delta_{\mathbf{u}_{1}} \cong U_{2} \Delta_{1}^{\prime} U_{2}^{T} \Delta_{E E^{T}} \mathbf{u}_{1}
$$

where

$$
\Delta_{1}^{\prime}=\left[\begin{array}{cc}
\xi & 0 \\
0 & \frac{1}{\lambda_{1}-\lambda_{3}}
\end{array}\right]
$$

The variable $\xi$ is defined as

$$
\begin{aligned}
\operatorname{if}\left(\frac{\lambda_{1}-\lambda_{2}}{\operatorname{mean}\left[\lambda_{1}, \lambda_{2}\right]}>\text { Tol }\right) \text { then } \xi & =\frac{1}{\lambda_{1}-\lambda_{2}} \\
\text { otherwise } & \xi=0,
\end{aligned}
$$


where Tol is a user defined tolerance. In experiments we use Tol $=10^{-6}$. The matrices $\Delta_{2}^{\prime}$, resp. $\Delta_{3}^{\prime}$, are defined similarly. Although this assistance looks quite curiously, it works very well as we will show in experiments.

Now, return to the question how we can estimate $C_{U}$, resp. $C_{V}$, having $C_{E}$. The equation (30) can be rewritten in matrix form as

$$
\delta_{\mathbf{u}_{1}}=U_{2} \Delta_{1}^{\prime} U_{2}^{T}\left[u_{11} I_{3}, u_{13} I_{3}, u_{13} I_{3}\right] \delta_{\mathbf{E E}^{\mathbf{T}}}
$$

where $\delta_{\mathbf{E E}^{\mathbf{T}}}$ is a vector rearranged from elements of the matrix $\Delta_{E E^{T}}$, equation (21), and $I_{3}$ is the $3 \times 3$ identity matrix. The error matrix of $E$ can be rearranged to [ error vector $\delta_{\mathbf{E}}$. From the equation (21) we can derive matrix $G_{E E^{T}}$ such that

$$
\delta_{\mathbf{E E}^{\mathbf{T}}}=G_{E E^{T}} \delta_{\mathbf{E}}
$$

Introducing new matrix $D_{\mathbf{u}_{1}}=U_{2} \Delta_{1}^{\prime} U_{2}^{T}\left[u_{11} I_{3}, u_{13} I_{3}, u_{13} I_{3}\right] G_{E E^{T}}$ the equation (33) can be rewritten as

$$
\delta_{\mathbf{u}_{1}}=D_{\mathbf{u}_{1}} \delta_{\mathbf{E}}
$$

Under the assumption of the zero mean noise for the covariance matrix of $\mathbf{u}_{1}$, we have

$$
C_{\mathbf{u}_{1}}=\mathrm{E}\left\{\delta_{\mathbf{u}_{1}} \delta_{\mathbf{u}_{1}}^{T}\right\}=D_{\mathbf{u}_{1}} \mathrm{E}\left\{\delta_{\mathbf{E}} \delta_{\mathbf{E}}^{T}\right\} D_{\mathbf{u}_{1}}^{T}=D_{\mathbf{u}_{1}} C_{E} D_{\mathbf{u}_{1}}^{T}
$$

The $9 \times 9$ covariance matrix $C_{U}$ is

$$
C_{U}=D_{U} C_{E} D_{U}^{T}
$$

where $D_{U}$ is the $9 \times 9$ matrix composed from the rows of $D_{\mathbf{u}_{\mathbf{i}}}$ matrices. A similar derivation can be done for $C_{V}$. The complete derivation can be found in [11].

Having $U, C_{U}$ and $V, C_{V}$, we can compute the mean values of Euler angles, $\overline{\mathcal{E}}$, resp. translation vector, $\overline{\mathbf{t}}$ from $(\bar{U}, \bar{V})$ using equations $(9,10)$. The covariances $C_{\mathcal{E}}$, resp. $C_{\mathbf{t}}$, can be estimated from $C_{U}$, resp. $C_{V}$, by the same way as in the equations $(13,14)$.

\section{Experiments}

In the previous section we have described how to estimate the uncertainty of the essential matrix, and consequently of the motion parameters. Several questions have arisen:

- How accurate is the analytic approach? Remember that we use the first order approximation of the error.

- Which calibration parameters are more important for different types of motion or for different types of a scene?

- What happens when the $F$ matrix is not exact, i.e. when there are some errors in the correspondences? 

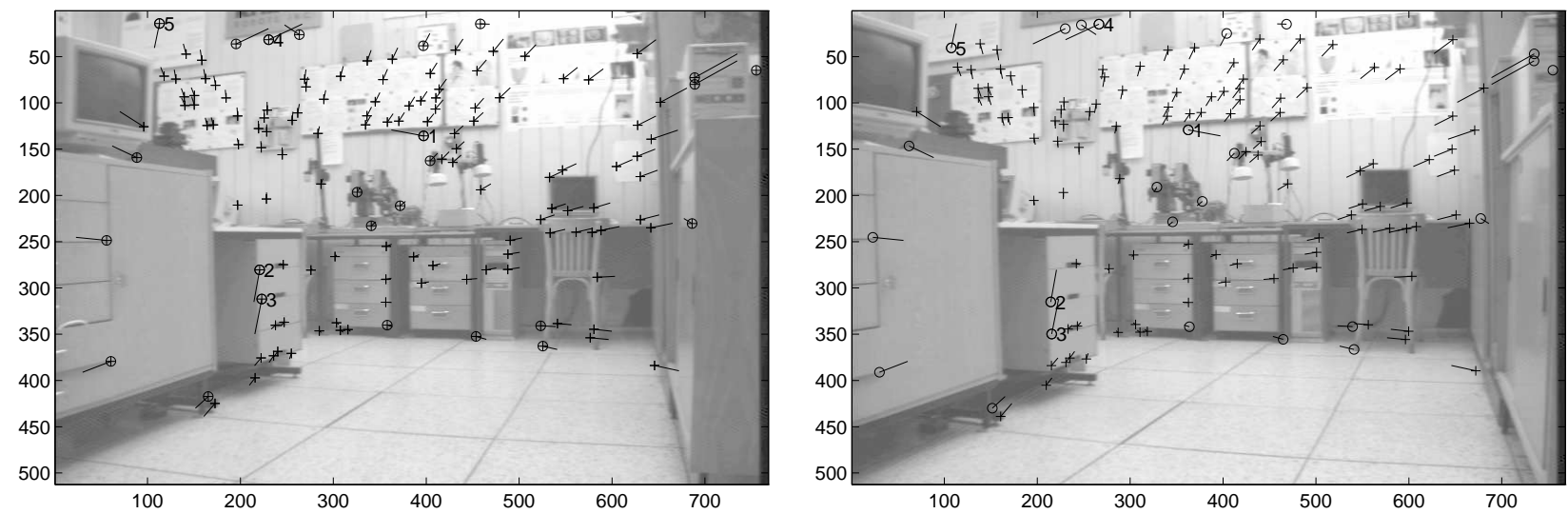

Figure 2: Image pair. Little crosses denote the correct correspondences, circles denote detected outliers and lines show the correspondence motion.

It seems obvious that different types of camera motion altogether with the arrangement of the scene affect the influence of the precision of the calibration parameters on the motion parameters estimation. The magnitude of the motion could also affect that relationship. Actually the influence of the inaccuracy in the calibration parameters depends on the motion of correspondences.

We did many tests with synthetic images, however in this section we focus on experiments with real images captured by the camera mounted on mobile robot. The robot undergoes planar motion and rotates around its vertical axis. We use the program by Z. Zhang [15] to find correspondences and the program by Svoboda and Pajdla [10] to detect outliers (false matches). For estimation of the motion parameters, we use linear method with point normalization [5]. Two consequent images with correct matches, outliers and motion of correspondences are in the Figure 2.

We add the artificial Gaussian noise to camera calibration parameters, i.e. elements of the matrix $K$, see equation (4). We have done many experiment with different types of motion and with different character of the errors in the calibration. To simulate the errors in camera calibration we have issued from statistical observation presented in [2]. We have concentrated on the reliability of the estimated variance in motion parameters w.r.t statistical observation.

There will be six lines on each graph. Straight lines denote the linear estimates of the variances of translation vectors resp. Euler angles. The fractional lines with little crosses denote statistical data. There are also three vectors on the left upper corners: (a) whatCalPar indicates what calibration parameters are noisy. (b) corrcoef indicates the correlation coefficients between noise in the calibration parameters, the significant is the first which denotes the correlation between $\alpha_{u}$ and $\alpha_{v}$. (c) t or Euls indicate the normalized translation vector resp. Euler angles.

The results belonging to the image pair from the Figure 2 are shown in Figure 3. The good reliability of the linear estimate w.r.t. statistical data can be observed up to $20 \%$ noise 
in the camera calibration parameters.
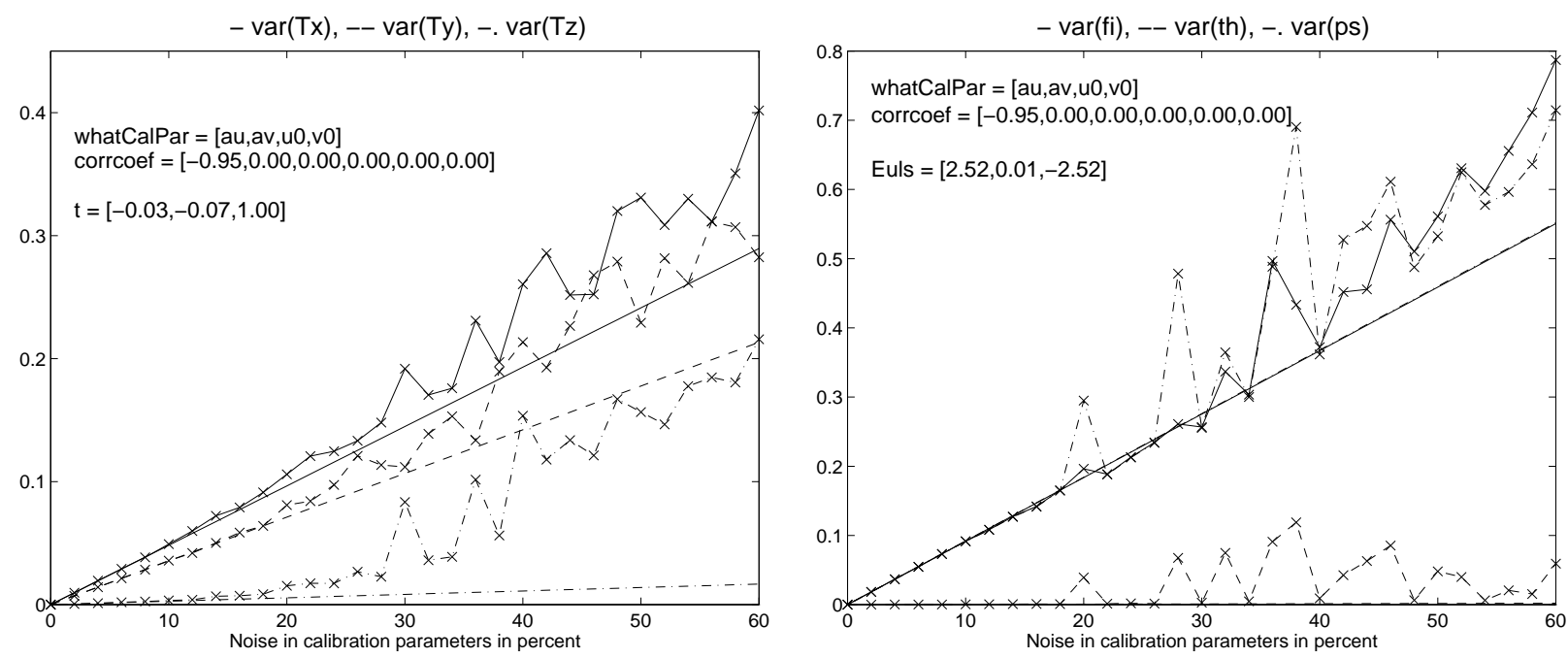

Figure 3: Variance in translation (left) and Euler angles (right). Lines with little crosses denote the statistical observation.

Two graphs in the Figure 4 illustrate that the noise in the horizontal calibration parameters $\alpha_{u}$ and $u_{0}$ affect mainly the horizontal part of the translation vector, $\mathbf{t}_{x}$. The similar relationship can be found between vertical parameters $\alpha_{v}$ resp. $v_{0}$ and the vertical direction of translation, $\mathbf{t}_{v}$. Since the robot rotates only around its vertical axis no essential relationships between uncertainty in calibration and Euler angles can be observed alike the translation.

\section{Conclusions}

We have presented an algorithm for the estimation of the variances of the camera motion parameters. We can characterize the uncertainty in the calibration parameters by their covariance matrix. We used the first order approximation to linearize the nonlinear relationship between the calibration parameters and the motion parameters. We have found that the accuracy in the "horizontal" calibration parameters, $\alpha_{u}$ and $u_{0}$, affects mainly the "horizontal" part of the translation, $\mathbf{t}_{x}$. We have observed similar relationship for the "vertical" parameters, $\alpha_{v}, v_{0}, \mathbf{t}_{y}$. The linear estimate was observed stable and credible up to $20 \%$ noise in the camera calibration parameter and even with noise in correspondences.

\section{References}

[1] Martin Armstrong, Andrew Zisserman, and Richard Hartley. Self-calibration from image triplets. In Bernard Buxton and Roberto Cippola, editors, 4th European Conference on Computer Vision 1996, pages 1-16. Springer-Verlag, LNCS 1064, April 1996. 

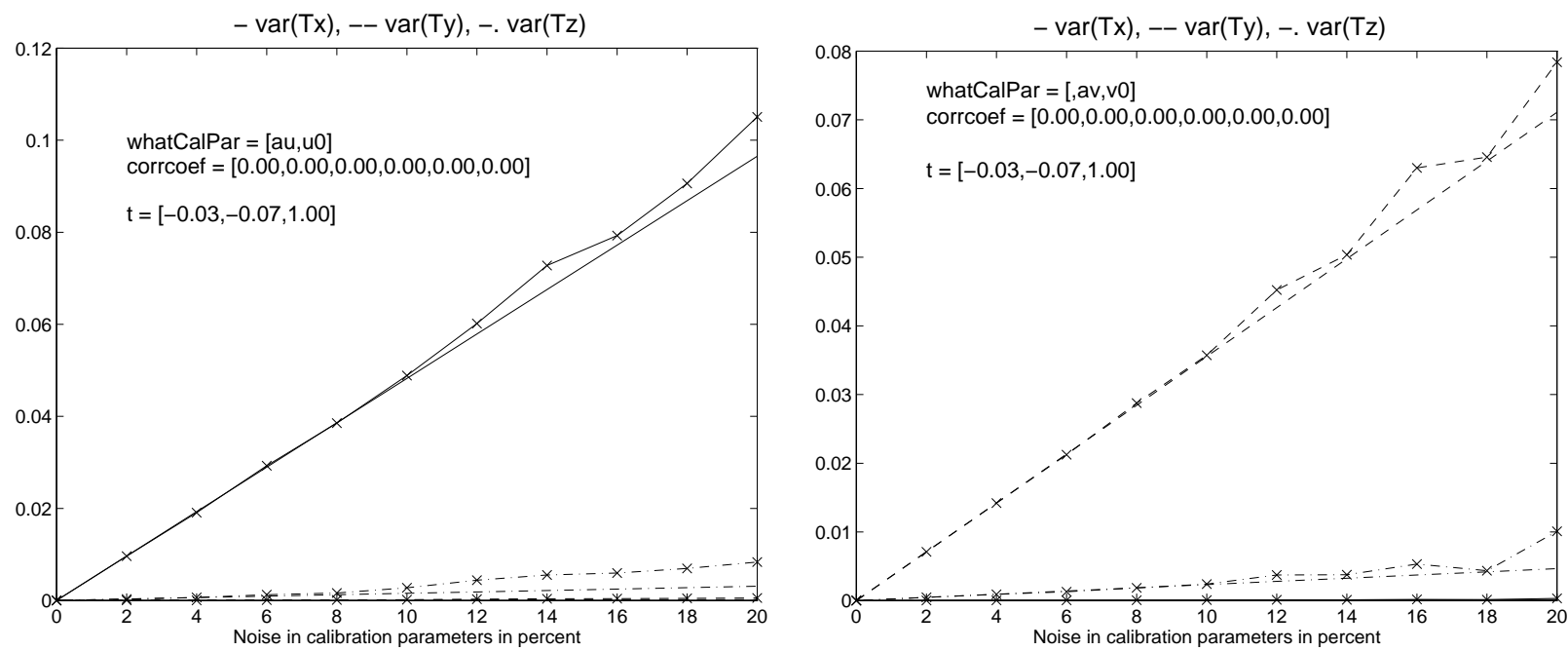

Figure 4: Variance in translation when the horizontal calibration parameters are noisy (left) and when the vertical calibration parameters are noisy (right). Lines with little crosses denote the statistical observation

[2] Giannoula Florou and Roger Mohr. What accuracy for 3D measurement with cameras? In International Conference on Pattern Recognition 1996, pages 354-358, Los Alamitos, California, August 1996. IEEE Computer Society Press.

[3] R.I. Hartley. Euclidean reconstruction from uncalibrated views. In DARPA-ESPIRIT workshop on Applications of Invariance in Computer Vision, pages 187-202, October 1993.

[4] Richard I. Hartley. Estimation of relative camera positions for uncalibrated cameras. In 2nd European Conference on Computer Vision, pages 579-587. Springer - Verlag, LNCS 588, May 1992.

[5] Richard I. Hartley. In defence of the 8-point algorithm. In Fifth International Conference on Computer Vision, pages 1064 - 1070. IEEE Copmuter Society Press, 1995.

[6] Roger A. Horn and Charles R. Johnson. Matrix Analysis. Cambridge University Press, 1985. 1987 reprinted with corrections, 1988,1990,1991,1992,1993.

[7] H.C. Longuett-Higgins. A computer algorithm for reconstruction a scene from two projections. Nature, 293:133-135, 1981.

[8] Quang-Tuan Luong, Rachid Deriche, Olivier Faugeras, and Theo Papadopoulo. On determining the fundamental matrix: Analysis of different methods and experimental results. Research report 1894, INRIA, April 1993.

[9] Stephen J. Maybank and Olivier D. Faugeras. A theory of self-calibration of a moving camera. International Journal of Computer Vision, 8(2):123 - 151, April 1992. 
[10] T. Svoboda and T. Pajdla. Eficient motion analysis. Research report K335/95/95, Czech Technical University, Facutly of Electrical Engineering, October 1995. 29 pages. Available at ftp://cmp.felk.cvut.cz/pub/cvl/ARTICLES/SVOBODA.

[11] T. Svoboda and P. Sturm. What can be done with a badly calibrated camera in egomotion analysis? Research report CTU-CMP-1996-01, Czech Technical University, Facutly of Electrical Engineering, Center for Machine Perception, September 1996. Available at ftp://cmp.felk.cvut.cz/pub/cvl/ARTICLES/SVOBODA.

[12] Roger Y. Tsai. A versatile camera calibration technique for high-accurancy 3D machine vision metrology using off-the-shelf cameras and lenses. IEEE Journal of Robotics and Automation, RA-3(4):323 - 344, August 1987.

[13] Juyang Weng, Thomas S. Huang, and Ahuja Narendra. Motion and structure from two perspective views: Algorithms, error analysis, and error estimation. IEEE Transactions on Pattern Analysis and Machine Inteligence, 11(5):451-476, May 1989.

[14] J.H. Wilkinson. The Algebraic Eigenvalue Problem. Oxford University Press, 1965.

[15] Zhengyou Zhang, Rachid Deriche, Olivier Faugeras, and Quang-Tuang Luog. A robust technique for matching two uncalibrated images through the recovery of the unknown epipolar geometry. Research report RR-2273, INRIA, May 1994. 